\title{
Do out-of-office laboratory tests affect diagnoses in general practice?
}

\author{
Joost OM Zaat ${ }^{1}$, François G Schellevis ${ }^{1}$, Jacques ThM van Eijk ${ }^{1}$ and \\ Koos van der Velden ${ }^{3}$ \\ 1 Department of General Practice and Nursing Home Medicine, Institute for Research in Extramural \\ Medicine, Vrije Universiteit, Amsterdam, ${ }^{2}$ Netherlands Institute of Primary Health Care (NIVEL), \\ Utrecht, The Netherlands.
}

\begin{abstract}
Zaat JOM, Schellevis FG, van Eijk JThM, van der Velden K. Do out-of-office laboratory tests affect diagnoses in general practice? Scand J Prim Health Care 1995:13;46-51.
\end{abstract}

Objective - To find out whether the GP diagnosis changed by out-of-office laboratory test results and whether his diagnosis became more certain.

Design - Descriptive study.

Setting - Dutch survey of morbidity and interventions in general practice: stratified random sample of $161 \mathrm{GPs}$ with a total list of 335000 patients.

Subjects - 2081 episodes of illness with at least one consultation with clinical chemistry, haematology, or serology tests and at least one follow-up consultation.

Main outcome measurements - Change in ICPC component or chapter between the consultation in which a laboratory test was ordered and the follow up contact; change in exact ICPC code in cases with important diseases (infectious diseases, haematological disorders, endocrine abnormalities, auto-immune processes and malignancies $(n=330)$ ); change in certainty of a diagnosis and change in somatic/ psychosocial orientation.

Results - After laboratory tests done in the first consultation the ICPC component changed in $46 \%$ of the diagnoses. Of the diagnoses made in first consultations without laboratory tests $\mathbf{4 1 \%}$ changed in the follow up consultation. The diagnosis after laboratory tests was the same as before in $51 \%$ of the consultations with important diseases. Certainty about a diagnosis increased significantly after laboratory tests $(p<0.001)$. An abnormal laboratory result did not affect the clinical certainty of the general practitioner or the percentage of altered diagnoses.

Conclusion - The usefulness of tests should be assessed not only in terms of the number of diagnoses changed or of the percentage of abnormal results, but also in terms of the changed certainty concerning a diagnosis.

Key words: laboratory test use, diagnostic process, general practice.

JOM Zaat MD, PhD, EMGO Institute/ Department of General Practice and Nursing Home Medicine, Vrije Universiteit, Van de Boechorststraat 7, 1081 BT Amsterdam, The Netherlands.

Dutch GPs are traditionally restrictive in their use of laboratory tests. They order one or more tests in only $4 \%$ of all patient consultations (1), while practitioners in Belgium order tests in $7 \%$ of their consultations (2), and family physicians in the US in $28 \%$ of all outpatient consultations (3). Little is 
known about the diagnostic impact of all these laboratory tests in everyday practice. In view of the role of laboratory tests in general practice we wanted to know if diagnoses change or gain in certainty after test use. We analysed, in a descriptive study, the data from the Dutch National Survey of Morbidity and Interventions in General Practice (4) in order to answer the following questions:

- how often did the diagnosis of the GP change after laboratory tests and how often could this have happened due to results of laboratory tests?

- did the certainty about his diagnosis change after receiving the results of laboratory tests?

- did the opinion of the GP conceming the somatic or psycho-social background of the problem change due to laboratory tests?

- was a change in diagnosis influenced when one or more tests gave abnormal test results?

\section{Methods}

\section{Design}

The design and data collection of the Dutch National Survey are described elsewhere (5). A random stratified sample of 161 general practitioners working in 103 practices with a total list of 335000 patients was drawn from all 6400 Dutch GPs. Data were recorded for all patient consultations (both surgery consultations and home visits) during three consecutive months between 1 April 1987 and 31 March 1988.

We selected for this study a group of illness episodes that met the following criteria:

- episodes with two or more consultations, haematological, clinical-chemical, or serological laboratory tests being performed in at least one consultation;

- at least one follow-up consultation after the one in which tests were performed;

- the first consultation had to be for presentation of a new or recurrent problem to the GP. Recurrent problems were defined as old problems that were not active at the start of the registration period.

The diagnostic 'impact' of a laboratory test early in an episode can be different from test use in later consultations. A distinction was therefore made between episodes with tests performed during the first consultation ('consultations with laboratory tests'), and episodes in which tests were performed during the second or later consultation ('consultations without laboratory tests').

\section{Measuring instruments}

All diagnoses by the GP were centrally coded according to an adapted version of the ICPC by specially trained research assistants (6). The ICPC chapters 'eye' (F), 'ear' (H), 'nervous system' , 'skin' (S), 'urology' (U), 'pregnancy' (W), disorders of the female sex organs' (X), and 'disorders of the male sex organs' ( $Y$ ) were combined into the category 'others' because these chapters covered only a small number of episodes with laboratory tests. Chapter $Z$ (social problems) has no separate diagnosis component and was therefore not included in the analysis.

Change of diagnosis was studied in two ways. In a global one we counted shifts between the component's symptoms (component 1) and diagnoses (component 7) within and between ICPC chapters as changes, disregarding shifts within these components. In order to investigate the changes in diagnoses in more detail (within the diagnosis component of an ICPC chapter) we selected the episodes with diagnoses in the second consultation for which clinical-chemical or haematological tests could have been important. The diagnoses selected in this second consultation were infectious diseases (with the exception of upper respiratory tract or lower urinary tract infections), haematological disorders, metabolic disturbances, autoimmune disease, and malignancy of the GI tract. Laboratory tests may be important to establish the diagnosis in such problems. We compared the diagnosis in this second consultation with the orginal diagnosis in the consultation before a laboratory test was done.

In each consultation the GP rated the certainty of his diagnosis on a five-point scale on the contact-registration form (very uncertain to very certain). Whether it was a somatic or psycho-social complaint was to be scored similarly (purely somatic, somatic with some psycho-social aspects, somatic with definitive psychosocial causes, psychosocial with some somatic aspects, pure pyscho-social). 
Results of all laboratory tests from hospital or general practice laboratories were classified as normal or abnormal, based on the reference values of each particular laboratory.

\section{Analysis}

Non-parametric tests for paired observations (Wilcoxon) were used to test differences in certainty and opinions concerning somatic causes of complaints between the consultations. A significance level of $5 \%$ was chosen.

\section{Results}

The events in 1857 first consultations with laboratory tests and their following second consultations were analysed, while 1322 episodes without laboratory tests in the first consultation were analysed.

\section{Change of diagnosis}

The diagnosis changed to another ICPC component in $46 \%$ of the 1857 first consultations with tests. The percentage of changed diagnoses varied considerably between ICPC chapters (Table I).
The component showing the greatest shift (73\%) was component 1, 'symptoms' from chapter A (General). The yield from laboratory tests in this group seems high, but of the 152 patients whose diagnoses from the first consultation belonged to this component, 41 had no other diagnosis in the second consultation; 20 diagnoses moved to 'psychic disorders' (10 to the symptoms and 10 to the diagnoses component, e.g. depressive state); 11 diagnoses moved to the diagnoses in chapter $B$ 'blood' (anaemia, etc.); 6 to endocrine diagnoses, 10 to the diagnoses in chapter $R$, 'respiration' (mainly upper airways infections); and the others were distributed over the remaining ICPC chapters.

The diagnosis changed in the follow-up consultation of $41 \%$ of the 1,322 first consultations without laboratory tests (Table 1 ). The percentage of changed diagnoses also varied considerably.

The important diagnoses (infectious diseases, malignancy of the GI tract, autoimmune disease, metabolic or hematological disorders) were established in 330 second consultations (18\%). Of these, $51 \%$ had the same ICPC code in both consultations. Laboratory tests may therefore have supplied important new information in half of these cases, i.e. in $9 \%$ of the total number of diagnoses.

Table I. Percentages of changed diagnoses in consultations with and without laboratory tests requested in the first consultation.

\begin{tabular}{llcc}
\hline & & WTTH TESTS & WITHOUT TESTS \\
\hline ICPC & $\begin{array}{l}\text { Diagnosis in } \\
\text { consultation }\end{array}$ & $\begin{array}{c}\text { Changed diagnoses in 2nd } \\
\text { 2nd consultation }\end{array}$ & $\begin{array}{c}\text { Changed diagnoses in } \\
\text { 2nd consultation }\end{array}$ \\
\hline A-1 & symptoms general & $73(152)^{*}$ & $68(41)$ \\
A-7 & diagnoses general & $52(160)$ & $58(79)$ \\
B-7 & diagnoses haematology & $36(110)$ & $47(17)$ \\
D-7 & diagnoses gastroenterology & $36(165)$ & $34(119)$ \\
K-7 & diagnoses cardiovascular & $37(108)$ & $31(55)$ \\
L-7 & diagnoses musculo-skeletal & $36(94)$ & $33(82)$ \\
P-1 & symptoms psychic & $65(91)$ & $61(59)$ \\
P-7 & diagnoses psychic & $48(127)$ & $36(45)$ \\
R-7 & diagnoses respiratory & $33(178)$ & $29(314)$ \\
T-7 & diagnoses endocrinological & $31(134)$ & $30(20)$ \\
& Others & $34(538)$ & $26(491)$ \\
\hline & Total & $46(1857)$ & $41(1322)$ \\
\hline
\end{tabular}

\footnotetext{
- In parentheses: the total number of diagnoses made during the first consultation for that particular category
} 
Table II. Percentages of certain and uncertain diagnoses in first consultations with laboratory tests (absolute numbers)

\begin{tabular}{lcccc}
\hline $\begin{array}{l}\text { Diagnosis 1st } \\
\text { conconsultation }\end{array}$ & Uncertain & Doubtful & Certain & $\begin{array}{c}\text { Total 2nd } \\
\text { consultation }\end{array}$ \\
$\begin{array}{lcccc}\text { Diagnosis 2nd } \\
\text { consultation } \downarrow\end{array}$ & & & & \\
\hline Uncertain & 25 & 8 & 4 & $(117)$ \\
Doubtful & 24 & 26 & 9 & $(222)$ \\
Certain & 51 & 66 & 88 & $(1023)$ \\
\hline Total lst consultation & 100 & 100 & 100 & \\
& $(220)$ & $(416)$ & $(726)$ & $(1326)$ \\
\hline
\end{tabular}

Changes in certainty and somatic/psychic score

Certainty concerning the diagnosis increased after laboratory tests (Table II). Only one quarter of the 220 uncertain diagnoses in the first consultation with laboratory tests remained uncertain in the second one; the diagnoses were now certain in somewhat more than half the cases $(\mathrm{p}<0.001)$. In contrast with this gain in certainty, the diagnoses in the consultations without tests became less certain during the second consultation than they were in the first one $(p<0.001)$.

With respect to the somato-psychic scale the group with laboratory tests showed a tendency to consider diagnoses as more somatic in the second consultation but this was not significant $(\mathrm{p}=0.21)$ (Table III). GPs considered the diagnoses to be somatic more often in first consultations without laboratory tests than in consultations with laboratory tests $(65 \%$ vs $55 \%)$.

\section{Abnormal test results}

There were $1,110(16 \%)$ abnormal test results out of 7,176 tests. In 603 consultations ( $32 \%$ of the consultations) the GP received one or more abnormal result(s). After abnormal tests, $44 \%$ of diagnoses changed, versus $43 \%$ after normal results.

Certainty about the diagnoses increased after laboratory tests for consultations with abnormal and with normal results (both $\mathrm{p}<0.001$ ). More diagnoses were generally considered to be somatic when test results were abnormal than when they were normal $(72 \%$ vs $57 \%, \mathrm{p}=0.001)$.

\section{Discussion}

There are few studies of the diagnostic impact of laboratory tests. In a hospital population, laboratory tests led to a change of diagnosis in $22 \%$ of consultations and to a 'better understanding' of the illness in $20 \%$ (7). Other studies also showed that only a small percentage of the problems with diagnoses was affected by supplementary diagnostic tests $(8,9)$. In general practice, for example, the erythrocyte sedimentation rate appeared to be mainly a tool for confirmation of a diagnosis, rather than a test for detection of unexpected pathology $(10,11)$.

The results of our study show that more than half the diagnoses did not change with respect to their ICPC component after receiving test results. The fact that the diagnosis did change in $46 \%$ of the consultations can probably not be considered to be (entirely) due to the tests performed. It made little difference whether a result was abnormal; moreover, similar percentages of diagnoses of consultations without tests also changed in the course of follow-up consultations. The 'net' profit from tests generally appears to be small: the diagnosis was clear in the first consultation in half the cases of 'important diagnoses'. Laboratory tests apparently served to confirm the diagnosis.

Some investigators consider that the desire for certainty is the main reason for the use of many and unnecessary tests (12). There are indications that lack of certainty as a characteristic of physicians is related to the use of laboratory tests (13-15). Comments such as "general practitioners feel more assured after laboratory tests" cannot be made on the basis of the data described here. We 
Table III. Opinion concerning somatic or psychic background of the diagnoses after the 1st and 2nd consultation in first consultations with laboratory tests (absolute numbers in parentheses)

\begin{tabular}{lcccc}
\hline $\begin{array}{l}\text { Diagnosis } \\
\text { 1st consultation } \rightarrow\end{array}$ & Somatic & $\begin{array}{l}\text { Somatic/ } \\
\text { psychic }\end{array}$ & Psychic & $\begin{array}{c}\text { Total 2nd } \\
\text { consultation }\end{array}$ \\
$\begin{array}{l}\text { Diagnosis } \\
\text { 2nd consultation } \downarrow\end{array}$ & & & & \\
\hline Somatic & 87 & 42 & 25 & $(1063)$ \\
Somatic/psychic & 9 & 32 & 22 & $(228)$ \\
Psychic & 4 & 26 & 53 & $(218)$ \\
\hline Total in & 100 & 100 & 100 & \\
1st consultation & $(1030)$ & $(293)$ & $(186)$ & $(1509)$ \\
\hline
\end{tabular}

can only state that the diagnosis in the second consultation was more certain after laboratory testing. Whether this certainty was correct or influenced patient care we cannot say.

However, our approach is not without problems. Laboratory tests are only a small part of the diagnostic process and it is difficult with our approach to decide whether a test result indeed contributed to a change of a diagnosis. Various other factors can also have contributed to this change. Our study does not include detailed information about either history-taking or physical examination. The question about the contribution of test results therefore cannot be answered conclusively.

It is possible that the GP consciously adapts the diagnosis in a consultation to the desire to carry out an investigation (16): a diagnosis of iron-deficiency anaemia may not be truly considered, but nevertheless the physician requests a haemoglobin estimation, and therefore diagnoses anaemia with a high degree of uncertainty - prior to obtaining the result of the test. If the result is normal, the physician will return to the real, first diagnosis (e.g. fatigue or work problems). In this instance the recorded 'diagnosis' was changed, but in the physician's mind the diagnosis had remained the same. This bias could not be taken into account and could well be one of the explanations for changed diagnoses. However, our central coding process minimized at least the interdoctor variation in the registration of their diagnoses.

In general, the benefit from laboratory tests seems to be that diagnoses gain in certainty, while frequently not changing. There is a need for fur- ther studies of the value of tests in evaluating the assurance of physician and patient.

\section{Acknowledgement}

The Dutch National Survey on Morbidity and Interventions in General practice was supported by the Ministry of Welfare and Health and Cultural Affairs.

\section{References}

1. Kluijt I, Zaat JOM, van der Velden J, van Eijk J.ThM, Schellevis FG. The use of clinical-chemical, haematological, and serological laboratory tests in general practice. Huisarts Wet 1991; 34: 67-71. [In Dutch, summary in English]

2. De Maeseneer J. General practice; a survey. Gent: Rijksuniversiteit Gent, 1989 [in Dutch, summary in English]

3. Rosenblatt RA, Cherkim DC. The structure and content of family practice. Current status and future trends. J Fam Pract 1982; 15: 681-722.

4. Bensing JM, Foets $M$, van der Velden $J$, van der Zee J. The Dutch national survey of morbidity and Interventions in general practice. Huisarts Wet 1991; 34: 51-61. [in Dutch, summary in English]

5. Foets $M$, van der Velden J. Dutch national survey of morbidity and interventions in general practice. Summary of the study design. Utrecht: NIVEL, 1992.

6. Lamberts $H$, Woods $M$, eds. International Classification of primary care. Oxford: Oxford University Press, 1987.

7. Wertman BG, Sostrin SV, Pavlova Z, Lundberg GD. Why do physicians order laboratory tests? JAMA 1980; 243: 2080-2. 
8. Stilwell JA, Young D, Cunnington A. Evaluation of laboratory tests in hospitals. Ann Clin Biochem 1980; 17: 281-6.

9. Hampton R, Harrison MJG, Mitchell JRA, Prichard JS, Seymour C. Relative contributions of historytaking, physical examination, and laboratory investigation to diagnosis and management of medical outpatients. BMJ 1975; ii: 486-9.

10. Dinant GJ, Knottnerus JA, van Wersch JWJ. Discriminating ability of the erythrocyte sedimentation rate: a prospective study in general practice. $\mathrm{Br} J$ Gen Pract 1991; 41: 365-70.

11. Grønlie M, Hjortdahl P. The erythrocyte sedimentation rate; its use and usefulness in primary health care. Scand J Prim Health Care 1991; 9: 97-102.

12. Kassirer JP. Our stubborn quest for diagnostic certainty: a cause of excessive testing. $\mathrm{N}$ Engl J Med 1989; 320: 1489-92.
13. Epstein AM, Begg CB, McNeil BJ. The effect of physicians' training and personality on test ordering for ambulatory patients. Am J Public Health 1984; 74: 1271-3.

14. Ornstein SM, Markert GP, Johnson AH, Rust PF, Afrin LB. The effect of physician personality on laboratory test ordering for ambulatory patient. Med Care 1988; 26: 536-43.

15. Zaat JOM, van Eijk JThM. General practitioners' uncertainty, risk preference and their use of laboratory tests. Med Care 1992; 30: 846-54.

16. Howie JGR. Diagnosis: the Achilles heel. J R Coll Gen Pract 1972; 22: 310-5.

Received August 1993

Accepted May 1994 